\title{
Enquête
}

Archives de la revue Enquête

$8 \mid 1993$

Varia

\section{Quelle volupté que la peinture}

Maupassant critique d'art

\section{Alain Guillemin}

\section{(2) OpenEdition}

Journals

\section{Édition électronique}

URL : http://journals.openedition.org/enquete/178

DOI : 10.4000/enquete. 178

ISSN : 1953-809X

Éditeur :

Cercom, Éditions Parenthèses

\section{Édition imprimée}

Date de publication : 2 septembre 1993

Pagination : 121-137

Référence électronique

Alain Guillemin, "Quelle volupté que la peinture », Enquête [En ligne], 8 | 1993, mis en ligne le 09 juillet 2013, consulté le 02 mai 2019. URL : http://journals.openedition.org/enquete/178; DOI : 10.4000/ enquete. 178

Ce document a été généré automatiquement le 2 mai 2019. 


\title{
Quelle volupté que la peinture
}

\author{
Maupassant critique d'art
}

\author{
Alain Guillemin
}

Si j'avais le choix cependant entre les plus belles

créatures vivantes et la femme peinte du Titien que huit jours plus tard je revoyais dans la salle de la tribune à Florence, je prendrais la femme peinte

du Titien. Florence... m'attire encore presque sensuellement par cette image de femme couchée rêve prodigieux d'attrait charnel. Quand je songe à cette cité... m'apparaît soudain lumineusement au milieu des souvenirs qui jaillissent cette grande toile longue où se repose cette grande femme au geste impudique, nue et blonde, éveillée et calme.

GUY DE MAUPASSANT, La vie errante.

Comme l'affirme à juste titre Vladimir Biaggi, dans son introduction aux chroniques sur la peinture publiées par Balland, il convient de «ruiner un lieu commun tenace selon lequel Maupassant aurait eu en peinture... culture médiocre et mauvais goût ${ }^{1}$ ». Cette idée reçue, dont Edmond de Goncourt fut le fielleux propagandiste, a pu d'autant plus trouver preneur que Maupassant, à la différence de nombre d'écrivains du XIX ${ }^{e}$ siècle, tels que Beaudelaire, Théophile Gautier, Émile Zola ou Octave Mirbeau, ne peut en aucun cas être assimilé à un salonnier, à un critique professionnel. Plus qu'un amateur éclairé - le terme est faible - Guy de Maupassant est, selon l'expression de Georgette Tadros, un amateur passionné, dont on peut suivre la passion à travers toute l'œuvre ${ }^{2}$. Sur les 24 chroniques que Maupassant a consacrées à l'art, au sens le plus large du terme, 13 concernent à des degrés divers les arts graphiques ${ }^{3}$.

Il réserve d'autre part quelques pages à la peinture italienne dans La vie Errante. Enfin, l'univers des peintres est évoqué dans trois de ses romans, Bel Ami (1885), Notre Coeur (1889) et surtout Fort comme la mort (1889), dont un des personnages principaux est le peintre Bertin, ainsi que dans 5 nouvelles, «Les dimanches d'un bourgeois de Paris » 
(31 mai-16 août 1880, Le Gaulois), "Le Voleur » (21 juin 1882, Le Gil Blas), " Une Soirée » (21 septembre 1883, Le Gaulois), « Miss Harriel » (9 juillet 1883, Le Gaulois), « Le modèle » (17 décembre 1883, Le Gaulois).

3 Les goûts de Maupassant en matière de peinture, nous le verrons, sont éclectiques. Ce qui lui attire les éloges des critiques et des biographes quand il défend les impressionnistes, le blâme quand il affirme des penchants pour certains peintres académiques. À vrai dire, il ne mérite ni cet excès d'honneur, ni cette indignité. Familier des cercles artistiques, Guy de Maupassant est bien de son époque, la fin du XIX ${ }^{\mathrm{e}}$ siècle, qui voit coexister, plus harmonieusement qu'on ne le croit, des mondes de l'art très contrastés. Surtout, ce qui, sans doute a le plus choqué les amants du beau et les dévots des avant-gardes, il affiche sans pudeur un " plaisir de regardeur » ou se donne à voir " la multiplicité hétérogène et indéfinie » des composantes de sa perception esthétique ${ }^{4}$.

\section{Un familier des cercles artistiques}

4 Par l'intermédiaire de Flaubert, dès 1875, jeune employé au Ministère de la Marine, Guy de Maupassant entre en relation avec Zola et chaque semaine retrouve, rue Saint Georges, Paul Cézanne, Huysmans et le critique Duranty. À partir de 1877, lorsque Zola achète à Médan, près de Poissy, une maison de campagne, Maupassant demeure un hôte assidu. À Paris, au café « Le Rochefoucauld », au coin de la rue N. D. de la Joliette et de la rue La Rochefoucauld, il croise Gerôme, Cormon, Degas, Gustave Moreau, Fantin-Latour. Dans les années 80, il retrouve ce dernier au café Gerbois, en compagnie de Renoir, Monet, Sysley, Pissaro, Manet, Degas, Zola et Duranty. À Etretat, si l'on en croit Pierre Borel, il côtoie Courbet, Monet et Diaz ${ }^{5}$.

D'autre part, le succès venant, Maupassant devient l'hôte assidu de nombreux salons où il peut rencontrer des peintres. Chez Juliette Adam, directrice de la Nouvelle Revue, à qui il donne en 1881 "En famille », il rencontre Bonnat, Bastien-Lepage, Gervex, Rodin. Chez Marguerite et Georges Charpentier, l'éditeur, 11 rue de Grenoble, il retrouve le monde politique de gauche, Gambetta, Clemenceau, mais aussi Monet et Renoir dont le tableau «Madame Charpentier et ses enfants» (1878) aura pour décor le salon de Grenelle. Dans le salon de la Comtesse Cahen d'Anvers, femme du banquier, il noue une amitié durable avec le peintre Albert Cahen d'Anvers, frère de son hôtesse. Il fait partie avec son ami Gervex de la cour d'admirateurs de la Comtesse Potocka et côtoie, chez la Princesse Mathilda, Albert Besnard, Bonnat et Fromentin. En dehors de ces fréquentations mondaines, plus ou moins épisodiques, Maupassant noue une véritable amitié avec 5 peintres. Jean Beraud (1848-1935), peintre de scènes de rue et de sujets mondains, que Maupassant considère comme "le plus charmant des fantaisistes ", est cité dans "Une soirée » et dans Fort comme la mort et le financier Walter, personnage de Bel ami possède un tableau de lui. René Bilotte (1846-1914) élève de Fromentin, peintre de paysages, canote avec Maupassant. Antoine Guillemet (1847-1918), élève de Monet et de Corot est aussi un assidu de la Grenouillère et Maupassant, dans son salon de 1886, le classe parmi les maîtres incontestés. Mais c'est sans doute avec Henri Gervex (1852-1929) et son cousin germain Louis le Poittevin (1847-1901) que les liens sont les plus étroits. Ce dernier obtint au salon de 1886 une médaille de troisième classe pour «Etretat ». En 1886, il loue le rezde-chaussée de son hôtel particulier à Maupassant, avec lequel il entretient depuis quelques années une correspondance suivie. C'est au début des années 80 que Gervex devient un intime de Maupassant et il voyage avec lui en Provence et en Italie. Il réalise 
un portrait de l'écrivain et en raison de son éclatante carrière de peintre mondain, moins insignifiant qu'on ne l'a dit - il était ami de Degas et Renoir et fit entrer Claude Monet au salon $^{6}$-, il a probablement servi de modèle à Olivier Bertin, personnage principal de Fort comme la mort, décrit comme «le peintre chéri des Parisiens et des Parisiennes, l'interprète le plus adroit de leurs grâces et de leurs tournures ${ }^{7}$ ».

Riche de cette expérience mondaine, Maupassant peut croquer, le plus souvent férocement, des portraits de tous les protagonistes du monde de l'art, les peintres, les spectateurs du salon, mais aussi les critiques. Dans Fort comme la mort, il décrit en ces termes Monsieur de Musadieu, inspecteur des Beaux-Arts et oracle des salons aristocratiques :

Apte à tout faire, semblait-il, il parlait de tout avec un semblant de compétence attachant et une clarté de vulgarisateur qui le faisait fort apprécier des femmes du monde à qui il rendait le service d'un bazar roulant d'érudition [...] il était au mieux avec les cinq Académies [...] et prêtait à ses connaissances ainsi glanées, un tour aisé, clair et bon enfant, qui les rendait facile à comprendre comme les fabliaux scientifiques [...] Les peintres, avec qui ses fonctions le laissaient en rapport constant le blaguaient et le redoutaient ${ }^{8}$.

7 Les goûts de Maupassant en matière de peinture, de l'avis de tous ses commentateurs, sont éclectiques, encore convient-il d'éclairer les facettes de cet éclectisme. Maupassant n'ignore pas la peinture ancienne. Ses préférences vont à l'école flamande, Rubens et Rembrandt, et à l'école italienne, Raphaël, Léonard de Vinci, mais surtout aux Vénitiens du XVI ${ }^{e}$ siècle, Le Titien, Veronèse, Le Tintoret, ou du XVIII siècle, comme Tiepolo. C'est-àdire les maîtres du clair-obscur et les coloristes :

L'école italienne a enfanté des coloristes éclatants, toujours un peu durs bien qu'admirables, et l'École Flamande a engendré ces hommes prodigieux qui, dans les gradations d'une seule note, ont su mettre tout l'infini des nuances. Un bout d'étoffe peint par Rembrandt, deux tons voisins posés par la main de cet admirable maître, nous ont révélé que ce qu'on voyait noir ne l'est pas, et nous ont montré, dans ces noirs lumineux, plus de couleur, plus de richesse, plus de variété, plus d'inattendu, plus de charme captivant que dans les toiles éclatantes de Rubens'.

En ce qui concerne la peinture de son siècle, Maupassant exprime son admiration pour tous les grands maîtres, quelle que soit l'école. Aussi bien Ingres et Delacroix que Courbet, qu'il met en scène dans «La vie d'un paysagiste ", sans oublier Millet. Mais sa préférence va aux peintres de Barbizon, en particulier Corot, et aux impressionnistes avec une préférence marquée pour Monet. Il n'est pas douteux que Maupassant fait montre d'une véritable sensibilité impressionniste, dont l'on retrouve des traces dans toute son œuvre. Ainsi, dans La vie errante, il évoque en ces termes le lever du jour en Tunisie:

L'aurore est rose, d'un rose intense. Comment l'exprimer? Je dirai Saumonée si cette note était plus brillante. Vraiment nous manquons de notes pour faire passer devant les yeux toutes les combinaisons de tous. Notre regard, le regard moderne, sait voir la gamme infinie des nuances. Il distingue toutes les unions de couleurs entre elles, toutes les dégradations qu'elles subissent, toutes leurs modifications sans l'influence des voisinages, de la lumière, des ombres, des heures du jour. Et pour dire ces subtiles colorations nous avons seulement quelques mots ${ }^{10} \ldots$

9 En suivant Claude Monet à la recherche d'impressions, il nous rend sensible à la difficulté de peindre le plein soleil ou la pluie :

Je l'ai vu saisir ainsi une tombée étincelante de lumières sur la falaise blanche et la fixer à une coulée de tons jaunes qui rendaient étrangement le surprenant et fugitif effet de cet insaisissable et aveuglant éblouissement. Une autre fois, il prit à pleines mains une averse abattue sur la mer, et la jeta sur sa toile. Et c'était bien de la pluie 
qu'il avait peinte ainsi, rien que de la pluie voilant les vagues, les roches et le ciel, à peine distincts sous ce déluge ${ }^{11}$.

Maupassant, qu'il traduit ainsi dans le conte « La Maison Tellier» :

Des deux côtés de la route la campagne verte se déroulait. Les colzas en fleur mettaient de place en place une grande nappe jaune ondulante d'où s'élevait une saine et puissante odeur, une odeur pénétrante et douce portée très loin par le vent. Dans les seigles déjà grands, des bleuets montraient leurs petites têtes azurées que les femmes voulaient cueillir, mais M. Rivet refusa d'arrêter. Puis parfois, un champ tout entier semblait arrosé de sang tant les coquelicots l'avaient envahi. Et au milieu de ces plaines colorées ainsi par les fleurs de la terre, la carriole, qui paraissait porter elle-même un bouquet de fleurs aux teintes plus ardentes, passait au trot du cheval blanc, disparaissait derrière les grands arbres d'une ferme, pour reparaître au bout du feuillage et promener de nouveau à travers les récoltes jaunes et vertes, piquées de rouge ou de bleu, cette éclatante charretée de femmes qui fuyait sous le soleil ${ }^{12}$.

11 En dépit de ce goût affirmé pour la peinture de ses amis impressionnistes, Maupassant ne méprise pas pour autant la peinture dite « académique ». Léon Bonnat, Meissonier, Roche Grosse, Détaille, entre autres, et bien sûr son ami Gervex retiennent ses suffrages. S'en formaliser, comme on le fait souvent, serait injuste et anachronique. Injuste car, sans tomber dans les excès de Dali, il convient de regarder d'un œil nouveau les productions de ces peintres très divers, rassemblées plus ou moins arbitrairement sous l'appellation de «pompiers" et de ne pas tous les précipiter dans la mer de la médiocrité. Anachronique car à cette époque de coexistence de mondes de l'art antagonistes, nombre de critiques avertis se comportaient comme Maupassant. Ainsi, Alfred de Lostalot, chroniqueur attitré de la Gazette des Beaux-Arts, qui, dès 1876, plaide la cause des impressionnistes dans la sage Chronique des Arts, voit en Paul Baudry, peintre académique aujourd'hui ignoré, la plus haute personnalité de l'art contemporain. Léonce Bénédicte, critique réputé qui deviendra conservateur du musée du Luxembourg en 1895, et commissaire général des Beaux-Arts à l'Exposition Universelle de 1900, admirateur de Gustave Moreau, Fantin-Latour et Puvis de Chavanne, compréhensif à l'égard des impressionnistes, apprécie les scènes bretonnes de Charles Cotteret et les panneaux décoratifs d'Henri Martin. Enfin Roger Marx, critique d'art au quotidien de gauche Le Voltaire, ami et admirateur des Goncourt, inspecteur général adjoint des Musées départementaux, qui reconnaît dès 1889 la valeur de Cézanne et défend activement Toulouse-Lautrec, ne méprise pas pour autant tous les artistes qui exposent au Salon ${ }^{13}$.

\section{Maupassant au Salon}

Guy de Maupassant s'est toujours refusé à devenir un salonnier. Dès 1880, Gervex lui propose d'écrire un salon en collaboration, mais il finit par se désister après avoir fait traîner les choses ${ }^{14}$. Il faut attendre 1886 , et ce sera la première et dernière fois, pour qu'il se livre, au profit du journal Le XIX ${ }^{e}$ siècle, fief d'Edmond About, à ce rituel du monde de l'art parisien. Ce qui constitue l'intérêt et l'originalité de ces cinq articles ce n'est pas la critique féroce de la peinture académique (en 1886, la scène artistique offre des spectacles très divers et l'art académique est largement battu en brèche, non seulement par l'impressionnisme, qui a trouvé droit de cité, mais encore par le symbolisme), mais le ton adopté par Maupassant. Il n'entre pas dans le temple de l'art pour célébrer gravement le 
beau ou dénoncer le laid avec véhémence, il étale ses plaisirs et ses déplaisirs et brocarde joyeusement, peintres, critiques, et visiteurs du salon.

Ayant introduit ses lecteurs "dans cette halle centrale de la peinture ${ }^{15}$ ", Maupassant commence par affirmer hautement que personne ne s'y connaît en peinture, ou du moins que personne n'est apte à porter un jugement impartial : les critiques, faute d'une science de l'exécution, les peintres, incapables de s'élever au-dessus de leurs préjugés d'école, les fonctionnaires des Beaux-Arts, ministre en tête, ignorants, le public, attiré par les grands formats et l'anecdote facile. À ce titre Maupassant met bien en évidence, dès 1886, ce que les historiens de l'art actuel constatent, les effets négatifs de la persistance du sujet, dans les années 1880-1890, en dépit des révolutions picturales. Alors que le peintre doit nous émouvoir "par son œuvre même et non par l'anecdote que son œuvre présente ${ }^{16}$ », les spectateurs du salon font l'inverse :

Le public va regarder les tableaux exactement comme les petits enfants regardent les images. Il s'intéresse d'abord aux sujets, cherche à comprendre l'aventure, s'inquiète ou s'amuse de la ressemblance des personnages avec des gens qu'il connaît. On s'écrie: - Tiens! Juliette, regarde donc si cette grosse femme ne ressemble pas à Mme Bafour ${ }^{17}$ !

Dans un deuxième temps, Maupassant pénètre avec nous dans le salon où les œuvres entassées pèle mêle, côte à côte, comme les marchandises d'un entrepôt [...] produisent d'atroces cacophonies de tons, des combats de rouge, des rencontres de bleus, des mêlées (innombrables) de couleurs exaspérées de se rencontrer ${ }^{18 .}$

Puis commence une description burlesque des œuvres qui, au fil des cinq articles, s'achève sur un souhait, la suppression du Ministère des Beaux-Arts, de l'aide de l'État et du Salon lui-même.

Maupassant répartit les peintres en deux courants et onze groupes sympathiques mais, là encore, il s'agit d'une parodie. Les critiques et les historiens d'art se sont efforcés, au XIX siècle, de trouver des systèmes de classement des peintres divers, voire antagonistes, en utilisant deux types de critères, les écoles (néoclassicisme, réalisme, romantisme) et les genres (portraits, paysages, scènes historiques, natures mortes, etc.).

Maupassant prend prétexte de cette pratique pour tourner en dérision, un homme

d'une profondeur d'esprit incroyable, connaisseur raffiné, autant qu'habile sousministre [...] inventeur des groupes sympathiques qui a divisé les peintres en deux courants, un courant ascendant un courant descendant, celui-ci faisant baisser, celui-là faisant monter le niveau sacré de l'art... les peintures militaires sont le courant qui fait monter, et les peintres de femmes nues le courant qui fait baisser ${ }^{19}$.

Il s'agit d'Edouard-Henri Turquet, sous secrétaire d'État aux Beaux-Arts depuis 1879, que Maupassant épinglera encore dans une chronique du Gil Blas, «Loi Morale », le 25 juin 1887:

Mr Turquet a tenté de donner aux artistes dévoyés une notion plus saine de l'art, de leur faire remplacer les cuisses nues des femmes par des culottes à troupiers et les poitrines fermes et bombées par des canons braqués pour la défense de la patrie ${ }^{20}$.

Pour juger de l'écart à la norme, il n'est pas sans intérêt de confronter la manière de Maupassant au style des critiques patentés qui ont aussi couvert le Salon de 1886. Un des tableaux les plus appréciés du Salon de 1886 fut le «Justinien» de Benjamin Constant, élève de Cabanel, qui commença comme orientaliste avant de se consacrer avec succès au portrait. George Lafenestre, qui deviendra, en 1888, conservateur des peintures au musée du Louvre, manifeste aussi son admiration dans la Revue des deux Mondes : 
La solidité des marbres polis, des métaux reluisants, des étoffes somptueuses est, en effet, ce qui attire d'abord le pinceau vigoureux de M. Benjamin Constant. Nul n'excède comme lui à construire en belle matière un intérieur de salle silencieuse où la ferme épaisseur des lourdes teintures enveloppe d'une harmonie profonde et sourde le chatoiement des brocarts et des soies, le scintillement des bijoux et des armes. Ses représentations voluptueuses ou tragiques de harems orientaux, ont eu un succès méritée ${ }^{21}$.

Maupassant, lui, en rend compte en ces termes :

[...] M. Benjamin Constant nous présente un Justinien qui semble fort attristé du départ de Sarah Bernnardt pour l'Amérique. Que fait-il au milieu de ses ministres et conseillers, vêtus avec un luxe qu'on ne rencontre plus aujourd'hui, dans les cours les plus opulentes ${ }^{22}$.

19 À la fin $\mathrm{du} \mathrm{XIX}{ }^{\mathrm{e}}$ siècle, au salon, les scènes de la vie paysanne figurent parmi les genres reconnus, qui peuvent conduire les peintres au plus haut niveau de la hiérarchie académique, en appliquant à la figure paysanne les règles de la peinture d'histoire, notamment la monumentalité des formes. Millet et Courbet ont consacré ainsi la dignité des travailleurs de la terre.

Nombre de peintres, quelle que soit leur école donnent donc dans le genre paysan. En 1886, parmi beaucoup d'autres, le paysagiste Julien Dupré, animateur avec Théodore Rousseau de Barbizon, offre aux regards des visiteurs du Salon un tableau intitulé «Le ballon ", actuellement conservé par le Metropolitan Museum de New-York, dont J. Noulens rend compte en ces termes dans l'Annuaire du Salon 1886 :

Monsieur Dupré, dans Le Ballon où les faneuses interrompent leur besogne pour regarder un aérostat dans la rue, est demeuré carrément vigoureux et rigoureux envers la nature, en refusant selon son habitude toute concession à l'école nouvelle qui estompe les formes et les tons et les noie dans une fluidité grise ou violette. L'auteur du ballon s'est donc uniquement préoccupé de rendre brutalement son impression et de bien mettre en valeur les figures vivantes sur fond de paysage ${ }^{23}$.

Ce qui donne, sous la plume de Maupassant :

Chaque fois que je retourne au Salon, un étonnement me saisit devant les paysanneries. Et ils sont innombrables aujourd'hui, les paysans. Ils ont remplacé les Vénus et les Amours, que seul M. Bourguereau continue à préparer avec de la crème rose. Ils bêchent, ils sèment, ils labourent, ils hersent, ils fauchent, ils regardent même passer les ballons, les jolis paysans peints ${ }^{24}$.

21 Il importait, pour l'intelligence de la méthode de Maupassant, et pour le plaisir, de préciser les traits de son ironie. Mais en agissant de la sorte, Maupassant ne cède pas seulement aux facilités de la chronique, genre littéraire qui réclame de la verve et de la légèreté, il affirme aussi des convictions esthétiques, qu'il convient maintenant d'analyser plus précisément.

\title{
L'œil et ses plaisirs
}

\author{
On vit, on pense, on souffre, on est ému par le \\ regard. Celui qui sait sentir par l'œil éprouve, à \\ contempler les choses et les êtres, la même \\ jouissance aiguë, raffinée et profonde, que \\ l'homme à l'oreille délicate et nerveuse dont la \\ musique ravage le cœur. \\ GUY DE MAUPASSANT, Madame Panisse.
}


Tous les commentateurs de Maupassant soulignent, à juste titre, le rôle central du regard chez un créateur qui définit le talent en ces termes : «Il s'agit de regarder tout ce qu'on veut exprimer assez longtemps et avec assez d'attention pour en découvrir un aspect qui n'ait été vu et dit par personne ${ }^{25}$.» Aussi, est-ce tout naturellement que Maupassant applique cette acuité du regard à la contemplation de la peinture. Plus précisément, la qualité de l'œil est le fruit d'une longue patience qui permet de distinguer les nuances les plus subtiles, « un regard fin et exercé... en saisit les accords invisibles pour la foule, en note les innombrables et discrètes modulations ${ }^{26} "$.

Cette dextérité n'est pas à la portée du premier venu, « les hommes que la nature a doué pour être d'excellents juges... sont rares, perdus dans le nombre et leur voix n'est entendue que plus tard, bien plus tard ${ }^{27}$ ». Ainsi, pour Maupassant, qui défend, en face de l'art, la position d'un créateur plus que celle d'un critique, à côté de l'œil de l'artiste, il y a l'œil du rustre qui ne distingue rien et l'œil du bourgeois qui ne voit que l'anecdote et la surface des choses. C'est sans doute pour cette raison, que Maupassant ne se contente pas de regarder, ni même de se regarder regardant, mais entreprend encore de regarder les autres regarder. À ce titre, le tableau d'Henri Gervex, «Une séance du Jury de peinture, au salon des artistes français » (1883), conservé au Musée d'Orsay, est particulièrement apte à éclairer notre propos. Cette scène de genre d'un ami intime de Maupassant pourrait illustrer «Au salon» ou L'Euvre de Zola qui décrit en ces termes le travail du Jury :

Ces travaux du jury étaient une rude corvée, où Bongrand lui-même usait ses fortes jambes. Tous les jours, le travail se trouvait préparé par les gardiens, un interminable rang de grands tableaux posés à terre, appuyés contre la cimaise, fuyant à travers les salles du premier étage, faisant le tour entier du Palais, et, chaque après midi, dès une heure, les quarante, ayant à leur tête le président, armé d'une sonnette, recommençaient la même promenade, jusqu'à l'épuisement des lettres de l'alphabet. Les jugements étaient rendus debout [...] des discussions arrêtaient parfois les groupes, on se querellait pendant dix minutes...28

Si Maupassant, comme le Zola de L'Euvre, non seulement dans « Au salon » mais dans le roman Fort comme la mort, met en scène la foule des spectateurs, les peintres, les critiques et le tout-venant, ce n'est pas seulement pour amuser ses lecteurs par des scènes plaisantes, se distinguant en cela des autres salonniers, il ne se contente pas de décrire ou d'évoquer les œuvres, il prend aussi en compte leur réception. En ce sens, Maupassant est, avant la lettre, un disciple de Jean Molino, puisqu'il envisage simultanément la peinture, comme objet du monde et forme symbolique, comme objet produit par un créateur, comme objet reçu ou plutôt reproduit par un récepteur ${ }^{29}$. Le regard exercé de Maupassant ne s'apparente cependant pas à celui d'un froid expert, d'un érudit qui s'emploie à analyser les formes, il débouche sur le plaisir, tant il est vrai que «l'intelligence, aveugle et laborieuse ne peut rien savoir, car on ne peut rien comprendre, rien découvrir que par les sens... seuls intermédiaires entre l'Universelle Nature et Elle ${ }^{30}$ ». En effet, comme le met bien en évidence Georgette Tadros, Maupassant n'analyse l'art qu'en tant qu'il lui procure une impression agréable, il ne formule pas ses jugements en fonction d'une théorie de l'art, mais décrit la sensation qu'il éprouve en présence d'œuvres ${ }^{31}$.

L'impression sensorielle qu'il reçoit d'un tableau est donc prépondérante et dicte son jugement sur l'œuvre, ce qui contribue à éclairer le sens de son éclectisme. Précisons d'abord que cette primauté accordée à la jouissance par Maupassant, dans son commerce avec l'œuvre d'art, ne réduit pas cette dernière à une source de plaisir comme les autres. 
$\mathrm{Au}$ contraire, il distingue clairement le beau artistique du beau ou du laid dans la vie ordinaire :

Il ne faut pas confondre la sensation directe qu'un objet quelconque produit sur nos sens avec la sensation complète que nous donne un art représentant et interprétant cet objet. La chose la plus affreuse et la plus répugnante peut devenir admirable sous le pinceau ou sous la plume d'un grand artiste. Il serait long et superflu d'analyser ici cette double émotion, d'en marquer la nature et les origines différentes. Il suffit de la constater et de l'affirmer ${ }^{32}$.

En d'autres termes, le retentissement affectif suscité par la contemplation d'une œuvre n'est pas exclusif, chez Maupassant, d'un jugement de goût. À ce titre, les quelques lignes qu'il consacre à Tiepolo dans La vie errante sont exemplaires. Il souligne d'emblée «la séduction des nuances qui grisent sensuellement le regard ", mais resitue Tiepolo dans la peinture $d u \mathrm{XVIII}^{\mathrm{e}}$ siècle en le comparant à Watteau et Boucher, avant d'étayer son admiration sur des considérations plus techniques, « l'ingéniosité de ses compositions, l'imprévu puissant et solide de son dessin, la variété de son ornementation, la fraîcheur inaltérable et unique de son coloris ${ }^{33}$ ».

À l'inverse, la valeur artistique d'un tableau n'est pas mesurable à l'aune de l'intensité du plaisir ressenti. Les louanges que Maupassant adresse aux peintres académiques comme Bougereau, Jean-Paul Laurens, Bonnat, Roll, Carolus-Duran ou Jean Béraud, ne signifient pas qu'il les met sur le même plan que Le Titien, Rembrandt ou Monet. Ainsi, lorsque le financier Walter fait visiter à Georges Duroy, personnage central de Bel Ami, sa collection de peinture, le narrateur souligne le charme grivois du tableau de Jean Beraud, «Le haut et le bas ", qui met en scène " une jolie Parisienne montant l'escalier d'un tramway en marche » sous le regard concupiscent des messieurs assis sur les bancs ou debout sur la plate-forme ${ }^{34}$. Mais cette appréciation esthétique, ne permet pas de conclure qu'il s'agit d'une œuvre de valeur exceptionnelle. Sans négliger le fait qu'il est pour le moins périlleux, comme le font certains critiques, tel Albert Marne Schmidt, d'assimiler les goûts de Maupassant à ceux de ses personnages.

"Primum voluptas», telle pourrait donc être la maxime de Maupassant, critique d'art. La jouissance provoquée par la contemplation de l'œuvre d'art est en effet le principe qui donne cohérence à son mode de perception esthétique. Et, dans cette perspective, l'affect l'emporte sur l'analyse formelle, l'émotion sur la description. En ce sens, d'une certaine manière, les jugements esthétiques de Maupassant se ramènent à la constatation « qu'il n'y a pas de mots pour la peinture et que cela crève les yeux ${ }^{35} »$. Cependant, les choses sont moins simples qu'il n'y paraît. En effet, si nous suivons Jean-Claude Passeron, lecteur du Philèbe de Platon, il faut bien admettre que l'expérience esthétique est une réalité protéiforme, «mélange de composantes hétérogènes qui doit précisément à sa mixité, l'impression subjective de richesse insaisissable, le sentiment confus d'une toute puissance de la multiplicité en acte ${ }^{36} »$.

De l'avis même de l'auteur, ce qui importe ce n'est pas la liste provisoire et toujours perfectible de composantes de la perception esthétique, mais leur co-présence et leur hétérogénéité. Un certain nombre de conséquences en découlent en effet. L'amour de l'art ne peut plus être assimilé à sa forme la plus virtuose et la plus rarement attestée, le sentiment de la beauté, mêlé d'intérêts extrinsèques, est irréductible à un pur jugement de goût, même chez les érudits et les historiens d'art, les dosages multiples de ces composantes interprétatives autorisent l'attention distraite, « les pactes faibles, majeure partie des pratiques artistiques ${ }^{37} »$. 
Même si «le retentissement affectif lié à un univers fantasmatique» domine chez Maupassant, il se combine avec d'autres composantes : «prise en compte de la structure sensorielle du message», «connivence idéologique» avec les impressionnistes, « complicité culturelle » avec les peintres académiques, " proximité interactive » avec les artistes en tant que créateurs, etc. Mais ce phénomène n'a rien d'exceptionnel. On pourrait retrouver de multiples composantes, diversement hiérarchisées et articulées, dans les écrits de Beaudelaire, Émile Zola ou Octave Mirbeau, et il serait même judicieux d'en dresser une typologie. En revanche, ce qui constitue l'originalité des écrits sur l'art de Maupassant et contribue sans doute à expliquer, pour une large part, le dédain manifesté à leur égard par les critiques littéraires et les historiens d'art, c'est qu'il ne dissimule pas le "plaisir impur " que lui procure la peinture, derrière la rhétorique convenue de la critique d'art, et traite avec une joyeuse impertinence les gardiens sévères du jugement de goût.

\section{NOTES}

1. G. de Maupassant, Au salon. Chronique sur la peinture, Paris, Balland, 1993. Préface et notes de Vladimir Biaggi, p. 16-17.

2. G. Tadros, Guy de Maupassant amateur d'art, Thèse pour le doctorat d'université, Paris, Faculté des Lettres et Sciences humaines, 1968, dactyl., p. 232.

3. Ibid., p. 304. Il s'agit de chroniques publiées dans Le Gaulois ("Madame Pasca », 19 décembre 1880 ; «Maison d'artiste », 12 mars 1881 ; «Balançoires », 12 mai 1881 ; «Vengeance d'artiste », 20 février 1882), dans Le Gil Blas ("Note d'un démolisseur », 17 mai 1882 ; «Bibelots », 22 mars 1883 ; «Les amateurs d'artistes", 20 juin 1885 ; «Les juges », 7 juillet 1885 ; «La vie d'un paysagiste », 28 septembre 1886) et dans la revue Le XIX $X^{e}$ siècle ( 5 articles publiés sous le titre « Au salon », 30 avril, 2, 6, 10 et 18 mai 1886).

4. J.-C. Passeron, « L'œil et ses maîtres », in Les jolis paysans peints, Marseille, IMEREC, 1990, p. 113.

5. P. Borel, « Maupassant avant la gloire », Revue de France, sept.-oct. 1927, p. 38.

6. V. Biaggi, in G. de Maupassant, Au salon..., p. 161

7. G. de Maupassant, Fort comme la Mort, Paris, Folio-Gallimard, 1985, p. 44.

8. Ibid., p. 75 .

9. V. Biaggi, in G. de Maupassant, Au salon..., p. 79-80.

10. G. de Maupassant, La vie errante, Paris, Louis Conard, 1902, p. 190-191.

11. G. de Maupassant, « La vie d'un paysagiste », in Id., Au Salon..., p. 135.

12. G. de Maupassant, « La maison Tellier », in Id., Contes divers (1875-1880), Paris, Robert Laffont, 1988, p. 146.

13. J. P. Boulion et al., eds, La promenade du critique affluent. Anthologie de la critique d'art en France 1850-1914, Paris, Hazan, 1990, p. 150-161.

14. V. Biaggi, in G. de Maupassant, Au salon..., p. 9-10.

15. Au salon..., p. 75.

16. Ibid, p. 81 .

17. Ibid, p. 78.

18. Ibid, p. 88.

19. Ibid, p. 89. 
20. G. de Maupassant, Chroniques 3, Paris, 10/18, 1968, p. 306.

21. La Revue des Deux Mondes, mai-juin 1886, p. 590.

22. Au salon..., p. 93.

23. J. Noulens, in Annuaire du Salon $2^{e}$ année 1886, Artistes français et étrangers au Salon de 1886, Paris, E. Dentu, 1887, p. 116.

24. Au Salon, op.cit., p. 104.

25. G. de Maupassant, Le roman, préf. de Pierre et Jean, Paris, Albin Michel, Le livre de poche, 1984 , p. 30.

26. Au salon..., p. 78.

27. Au salon..., « Les juges », p. 64.

28. E. Zola, L'CEuvre, Paris, Le livre de poche, 1978, p. 329.

29. J. Molino, « Esquisse d'une sémiologie de la poésie », La petite revue philosophique, Montréal, G, 1, Aut. 1984, p. 5-12.

30. G. de Maupassant, La vie errante, p. 23.

31. G. Tadros, Guy de Maupassant amateur d'art, p. 232.

32. G. de Maupassant, Au salon..., « Les juges », p. 62.

33. G. de Maupassant, La vie errante, p. 251- 252.

34. G. de Maupassant, Bel Ami, Paris, Press-Pocket, 1990, p. 130.

35. P. Bonnefis, Comme Maupassant, Paris, Presses universitaires de Lille, 1981, p. 82.

36. J.-C. Passeron, «L'œil et ses maîtres », p. 115. les composantes de la perception esthétique, isolées par J.-C. Passeron, sont les suivantes: 1) Structure sensorielle du message, 2) Connivence idéologique, 3) Complicité culturelle, 4) Connaissance et reconnaissance des formes, 5) Légitimité sociale et culturelle, 6) Effet d'ancienneté ou de rareté de l'œuvre, 7) Proximité interactive (actuelle ou virtuelle) avec les artistes, 8) Gratification, ascétique ou non, des savoirs d'accompagnement, 9) Retentissement affectif, 10) Sentiment de correction et de conformité à un corpus de règles.

37. Ibid. 